\title{
Influences of Schooling and Urban-Rural Residence on Gender Differences in Cognitive Abilities and Academic Achievement ${ }^{1}$
}

\author{
Harold W. Stevenson, Chuansheng Chen, and James Booth \\ University of Michigan
}

A total of 1151 children from indigenous Quechua-speaking families residing in squatter settlements of the city and in two remote rural environments in Peru was given a battery of 16 tests of academic achievement and cognitive abilities. The former tests assessed reading and mathematics achievement and the latter tapped a broad range of cognitive functions. Children were enrolled in first, second, or third grade or did not attend school. "Younger" children were from 6 to 8 years old, and "older" children were from 9 to 12 years old. Large differences in cognitive functioning were associated with attendance at school, grade in school, age, and urban-rural residence. Gender was found to account for less than $5 \%$ of the variance in children's performance on cognitive and academic tasks. Gender effects appeared to decline with increased amount of schooling. This was reflected in interactions involving gender and schooling and in a greater number of significant gender effects for children who did not attend school or were in first grade. The results present a complicated picture of various interactional effects of task, location, age, and schooling on the detected gender differences in cognitive abilities and academic achievement.

Most psychological studies of gender differences in cognitive abilities have relied upon urban, schooled children and adults from industrialized countries as their subjects. Despite the long interest in the topic, we know

${ }^{1}$ The study was supported by grants from the National Science Foundation and the W. T. Grant Foundation. We are indebted to many people who participated in this project, especially Beatrice Bonnevaux, Alexander Wilkinson, Timothy Parker, Teofilo Altamirano, and Max Gonzales. We wish to thank the examiners, research assistants, parents, and children for their contribution to this research. 
very little about possible gender differences in cognitive abilities in other populations or in other cultures. Reports have most frequently concentrated on mathematics and reading (Hanna, 1988; Harnisch, 1986; Harnisch et al., 1986; Lummis \& Stevenson, 1990), and a few studies have compared other cognitive abilities of boys and girls in other industrialized countries (e.g., Stevenson et al., 1985). Studies of gender differences in cognitive abilities of individuals residing in Third World countries or of children who have not attended school are practically nonexistent.

Thus, when Linn and Hyde (1989) conclude that "gender differences on cognitive and psychosocial tasks are small and declining," they are basing their conclusions on conditions that exist primarily in one culture-the United States - and among members of that culture who are enrolled in secondary schools and colleges. Similarly, Hyde and Linn's (1988) conclusion that the "gender difference in verbal ability is currently so small that it can effectively be considered to be zero" (p. 64) is based on a meta-analysis of recent studies of schooled children in the United States and Canada. The same constraints existed within the studies reviewed by Hyde et al. (1990). As a consequence, it is nearly impossible to evaluate the generality of the phenomena they or others (e.g., Feingold, 1988) have detected. Indeed, Linn and Hyde (1989) recognize that even within the literature that does exist, the magnitude of gender differences reported is "clearly a function of context or situation."

It seems likely that variables such as cultures, residence in the city or in the countryside, and attendance at school would have strong influences on gender differences in cognitive development. These are the variables with which we are concerned in this report. Cultures differ greatly in the degree to which different roles are assigned to males and females and in the types of upbringing that exist in efforts to foster such differences. The tendency during recent decades for males and females in Western cultures to have increasingly common experiences is not necessarily the case in other parts of the world, especially in those that maintain beliefs about male and female inequality.

It also seems likely that residence in the city or in the countryside might influence the strength of gender differences in cognitive abilities. City life requires cognitive functions that are different from those necessary for daily life in remote rural villages. In the city, for example, individuals are frequently confronted with the need to read and to calculate. This is less likely to be the case in remote areas of the countryside, where communication occurs less frequently through the printed word and the economy relies on bartering rather than on monetary transactions. Depending upon parental expectations and the duties assigned to children, the demands made on cognitive abilities for boys and girls in the two environments may differ. 
The third influence on the course of cognitive development in which we are interested is schooling. Schooling is especially likely to influence gender differences among children in Third World countries, where children who do not attend school play a central role in the family's life and economy. They do this by caring for younger children, tending animals, and helping in the home and field. These experiences differ greatly from those of children who attend school. Arguments can be made both pro and con concerning the suggestion that schooling should heighten gender differences in cognitive functioning. Children who attend school are faced with the attitudes teachers often express about the abilities of boys and girls. We have found, for example, that American elementary school teachers express more favorable attitudes about girls than about boys in a broad range of cognitive and social characteristics (Stevenson et al., 1976). Moreover, elementary school teachers in Japan, Taiwan, and the United States held attitudes about abilities in reading and mathematics that differed for boys and girls (Lummis \& Stevenson, 1990). Such beliefs and attitudes of teachers may increase gender differences in cognitive functioning the longer children attend school. Conversely, it can be argued that the common experiences boys and girls receive through formal instruction should reduce gender differences in cognitive performance. During elementary school, boys and girls typically study the same subjects, have the same teachers, and share other common experiences at school.

The purpose of the present report is to extend our knowledge by analyzing gender differences among schooled and nonschooled children from several different environments with a battery of tasks that covered a broad spectrum of cognitive abilities. The study was conducted with children from Quechua-speaking Indian families, the predominant indigenous population of Peru. Many of these families do not enroll their children in school at the legally required age, thus it was possible to obtain samples of both nonschooled and schooled children.

The children lived in the shantytowns (barriadas) of Lima and in two rural environments: remote villages in the Peruvian highlands (sierra) and the tropical rain forest (selva). In addition to investigating the effects of schooling and environment of gender differences, it also was possible to evaluate the effects of chronological age. Some of the children attended first grade at or near the appropriate age and others delayed their entrance into school until they were over 9 years old.

There is no clear theoretical basis for hypothesizing the direction of the gender effects for schooled and nonschooled or for urban and rural children. It seemed likely, however, that the privileged status of boys, such as exists in traditional societies found in countries like Peru, would increase the likelihood of gender differences in cognitive development. Boys are 
allowed to have a greater range of experiences in the society outside the home than are girls, who are expected to remain at home and assist their mothers in child rearing and household duties. The breadth and diversity of the boys' experiences should facilitate their cognitive development, especially in the city, where they are expected to begin earning money for their families at an early age. Gender differences should be reduced, however, by the common experiences boys and girls share when they attend school. Similarly, gender differences should be decreased to the degree that families have abandoned aspects of their traditional culture in the process of adapting to city life. It was expected, therefore, that gender would interact significantly with schooling and environment in influencing children's scores on the tests of cognitive abilities and academic achievement.

\section{METHOD}

\section{Locations}

The two major ethnic groups in Peru are Quechua-speaking Amerindians and mestizos (mixed Indian and Spanish). We chose Quechua children primarily because of the ease of finding Quechua children who did not attend school. The Quechua families we studied are descendants of the Chancas, a group that resided in the region of Andahuaylas during the reign of the Incas. Biological tests have established the common genetic backgrounds of the sierra and selva groups (Frisancho et al., 1975). Thus, any differences found among children from the three locations cannot be attributed to differences in their genetic characteristics.

Lima. In the barren hills on the outskirts in Lima, millions of persons live in squatter settlements. The inhabitants of these settlements are primarily Quechua peasants who have migrated from the highlands in various waves throughout the past three decades. The residents of the barriada in which we conducted the study came primarily from the region of Andahuaylas. Migrants to Lima must learn Spanish in order to find employment, and all instruction in the schools is in Spanish. Nearly all children, but not all parents, were more fluent in Spanish than in Quechua. Families in the barriadas are very poor and neither parent typically has secure employment.

Andahuaylas. Families in Andahuaylas have lived in the highlands for many centuries. The primary language for all social interactions is Quechua and families in Andahuaylas retain many aspects of their traditional culture. Their major occupations are farming and herding. 
Lamas. Over $\mathbf{5 0 0}$ years ago a group of Chancas, defeated in battle by the Incas, moved hundred of miles northeast to Lamas, which is in the selva. These Quechua speakers have never intermarried with mestizos or other selva Indian groups. They are farmers and engage primarily in a barter economy. In order to find satisfactory land for farming, parents must leave their villages during the week for chacras, plots of land deep within the rain forest, returning only on weekends. Children typically remain in the villages, tended by their older siblings and a few village elders.

\section{Subjects}

Our sample included 1151 children: 387 from Lima, 428 from Andahuaylas, and 336 from Lamas. Among these, $51 \%$ were girls. Two groups of children were included: (a) 6-8 year olds ("younger children") who had not attended school or were enrolled in first grade and (b) 9-12 year olds ("older children") who had not attended school or were enrolled in first, second, or third grade. We included children from all of the schools we could find in each location. Our procedure for locating children who did not attend school was to send a person to the neighborhoods or villages. This "scout" sought out families with a child of the proper age and attempted to arrange for the child's participation in the study.

We had excellent cooperation in recruiting subjects and encountered practically no loss of subjects once testing began. The children seldom had books or toys and expressed great interest in being able to "play" with the colorful test materials in the presence of an attentive and responsive adult. The children were also given snacks and trinkets during the course of the testing in order to maintain their attention and cooperation. Because of the large number of tasks, testing lasted for several hours and was sometimes broken into two sessions.

\section{Measures of Achievement and Cognitive Ability}

Because there were no standardized achievement tests for reading and mathematics in Peru, we devised our own tests after reviewing textbooks used in Peruvian schools and discussing possible items with Peruvian colleagues. Since the language of instruction in Peruvian schools is Spanish, the reading test was written in Spanish. It consisted of reading letters and words, selecting pictures described by words, and reading comprehension. The mathematics test involved counting, reading numbers, and answering oral word problems and written computation problems. The reliability (alpha statistic) for the tests was high: .94 for reading and .91 for mathematics. 
In constructing the cognitive tests we sought to tap a broad range of cognitive skills, to include material that was relevant to the children's experiences, and to select tasks that could be easily administered. Instructions were written in both Quechua and Spanish so that the children could be tested in the language in which they were more skilled. Nearly all children in Lima and the majority of children in Lamas were tested in Spanish; nearly all children in Andahuaylas were tested in Quechua. There were both verbal and nonverbal forms for some of the tasks. In the verbal form, all information was conveyed by words; in the nonverbal form the examiner demonstrated what was to be done. No systematic interactive effects with gender were found for the verbal and nonverbal forms of the tasks, thus the data from the two forms are combined in the presentations of the data.

The cognitive tasks were given in 12 different orders to ensure that the level of performance on particular tasks would not be unduly influenced by such factors as the child's possible initial shyness and/or later fatigue. The achievement tests were always given after the cognitive tasks in order to avoid upsetting the children who did not attend school. We included demonstration trials whenever possible and repeated them until the children appeared to understand the procedure.

The cognitive tasks ranged from relatively easy ones involving recall of scenes to more complex ones testing children's understanding of concepts. Testing was discontinued in most tasks after the child missed a certain number of successive items, typically 3 or 4 . The following paragraphs contain brief descriptions of the tasks.

1. General Information. The test was similar to the general information tests commonly included in tests of intelligence. The answers to the questions were not necessarily taught directly in school, but depended upon information that could be absorbed from everyday experience. They were asked, for example, what pumps blood through their bodies and why blankets keep us warm.

2. Concept Formation. The concept formation task was presented in three steps. In each step the stimuli consisted of pictures of familiar fruits, flowers, and birds. In the first step the child learned a discrimination involving three of the stimuli: a fruit, a flower, and a bird. A new set of three stimuli was then introduced to test for transfer. In the third step, different sets of three stimuli were used to test whether the child had learned a generalized concept.

3. Series Completion. Series of line drawings depicting concepts such as number, size, and sequences of actions could be completed by placing items in a correct sequence. There was, for example, a set of drawings depicting a guinea pig in various stages of running behind a tree. Several 
drawings established the action or content of a series (e.g., a guinea pig approaching a tree) and the child had to place three items in the correct order to complete the series (e.g., the guinea pig crossing behind and proceeding beyond the tree).

4. Seriation. The seriation tasks were of the types commonly used in Piagetian studies; the child was required to organize an array of objects that differed according to one dimension, such as length, or arrays of objects that differed in two dimensions, such as color and number.

5. Perceptual Learning. A horizontal display of six $3 \times 4$ in blackand-white photographs of adults' hands was presented. The child was asked to match members of a deck of photographs with those in the array. Careful attention had to be paid to the distinctive features of each hand in order to make the correct matches. The procedure was repeated to assess the degree of learning that had occurred during the first trial.

6. Map Reading. An array of nine locations was defined by the pattern of which nine markers had been placed on the ground. The child was asked to place colored plastic shapes in the various locations. The child was guided by a "map" depicting the locations in which the plastic shapes were to be placed. The number of plastic shapes to be placed ranged from three to five.

7. Matrices. Patterns of colored dots arranged in a $6 \times 6$ matrix were shown to the child one at a time. The child had to reproduce each pattern from memory. The patterns ranged from a single vertical line of six dots of a single color to complex patterns involving several colors and the whole matrix. When the child demonstrated an inability to repeat a design from memory, the procedure was repeated and the child was asked to copy the designs.

8. Digit Span. Strings of numbers of increasing length were spoken by the examiner and repeated from memory by the child. Each child was tested with both a Quechua and Spanish version. The strings contained three, four, five, and six digits. Two strings of each length were presented. Results from the child's preferred language are included in this report.

9. Auditory Memory. The child was asked to repeat a series of taps demonstrated by the examiner. The taps were separated by short (S) and long (L) pauses (e.g., S-L-S-S-L). The series ranged from a simple pattern of three short pauses to ones involving up to 10 short and long pauses.

10. Story Memory. The child was read a short story about two children and was then asked 15 questions about what had been read, including the title of the story, the main characters, the central action, etc.

11. Memory for Movements. In one version of the task, sequences of bodily movements were demonstrated by the examiner and the child was asked to repeat them. In the second version, the movements were described rather than demonstrated by the examiner. From two to five movements, such as touching the nose and lifting a leg, were included in successive trials. 
12. Visual Memory. The child was shown a scene composed of an array of miniature animals, objects, and people. The scene was then covered with a cloth and the child was asked to recall the items that had been seen.

13. Memory for Pairs. The items used in the visual task were paired and the child was asked to repeat the pairings shown by the examiner. In a verbal version of the task the pairings were described by the examiner. For example, the child was shown a llama on a bridge and a girl on a path. The items were removed and the child was asked to reconstruct the pairs. The number of pairs presented in successive trials was two, four, and seven.

14. Memory for Location. Pictures of common objects were arranged in a linear array before the child and then turned over. The child was shown duplicates of each picture in a random order and was asked to point to its pair in the linear array. In a second version the pictures in each position were not shown, but were named. There were two sets of seven pictures.

It should be pointed out that data are not reported for several additional tasks because they proved to be either too easy or too difficult. Very few children apparently could understand the analogies or observational learning tasks we presented, for the average scores in all groups were low; on the other hand, children in all groups received a nearly perfect score on a matching task involving photographs, objects, and line drawings.

The reliability (alpha statistic) of the tasks was generally high: general information (.71), concept formation (.86), series completion (.71), seriation (.72), perceptual learning (.79), map reading (.81), matrices (.92), digit span (.86), auditory memory $(.84)$, story memory $(.71)$, memory for movements (.81), visual memory (.76), memory for pairs (.74), and memory for location (.72).

\section{Examiners}

Both male and female examiners were employed. No attempt was made to pair examiner and subject according to gender. The examiners were primarily residents of the regions in which the children lived, but in several cases it was possible for the examiners to work in two locations.

\section{RESULTS}

The design of this study allowed us to examine gender differences related to schooling versus nonschooling, amount of schooling, location, and chronological age. Each of the latter variables influenced the children's 
performance, but with the exception of the amount of schooling their interactions with gender were rare and unsystematic. Gender differences never accounted for more than $5 \%$ of the variance in the children's scores, while a combination of the other background variables typically accounted for more than $20 \%$. The analyses on which these conclusions are based are discussed in the following paragraphs.

The data for children who did not attend school or who attended first grade are presented in Table I in terms of mean percentage of items correct, standard deviations, and $d$ scores. Children's test scores differed greatly among the three locations. Children of Lima obtained the highest scores and the lowest scores were made by the children in the remote jungle region of Lamas. In terms of gender differences, the general tendency was for boys in Lima and Andahuaylas to obtain scores slightly higher than those of girls, or for the scores of boys and girls to be nearly identical. In Lamas, there were indications of a reversal of this trend, although none of the effects was statistically significant. These tendencies are evident in the fact that the vast majority of the $d$ scores for Lima and Andahuaylas were positive, while many of those of Lamas were negative. The $d$ scores serve as indicators of the size of the effects associated with gender, and were computed by subtracting the means of the girls from those of the boys, divided by the pooled within-gender standard deviation (Hedges $\&$ Becker, 1986). Also, in a series of 2 (gender) $\times 2$ (age) $\times 2$ (schooling) analyses of variance done separately for each location, there was a greater tendency for gender effects to be statistically significant in Lima and Andahuaylas than in Lamas. The significant effects are indicated in Table I. Nevertheless, in a similar series with location included as a fourth variable, there were frequent gender effects but few significant interactions (see Table II). The interaction terms that were significant represented complex interactions involving age and/or location and/or schooling.

The scores for the older children who were enrolled in first through third grades are summarized in Table III. The number of significant gender effects was much smaller than it was in the analysis of the performance of the children who never attended school or who were in the first grade (see Table II). Again, there were few significant interactions; in all cases, however, they reflected a moderating effect of schooling on differences between the scores of boys and girls. Specifically, there was a tendency for the gender differences to decrease with increasing years of schooling for Matrices, Story Memory, Memory for Movements, and Memory for Pairs.

Another index of the influence of gender on children's cognitive and achievement scores was obtained by determining the percentage of variance attributable to gender in the scores on each of the tasks. Background variables (dummy variables for location, schooling versus nonschooling, 


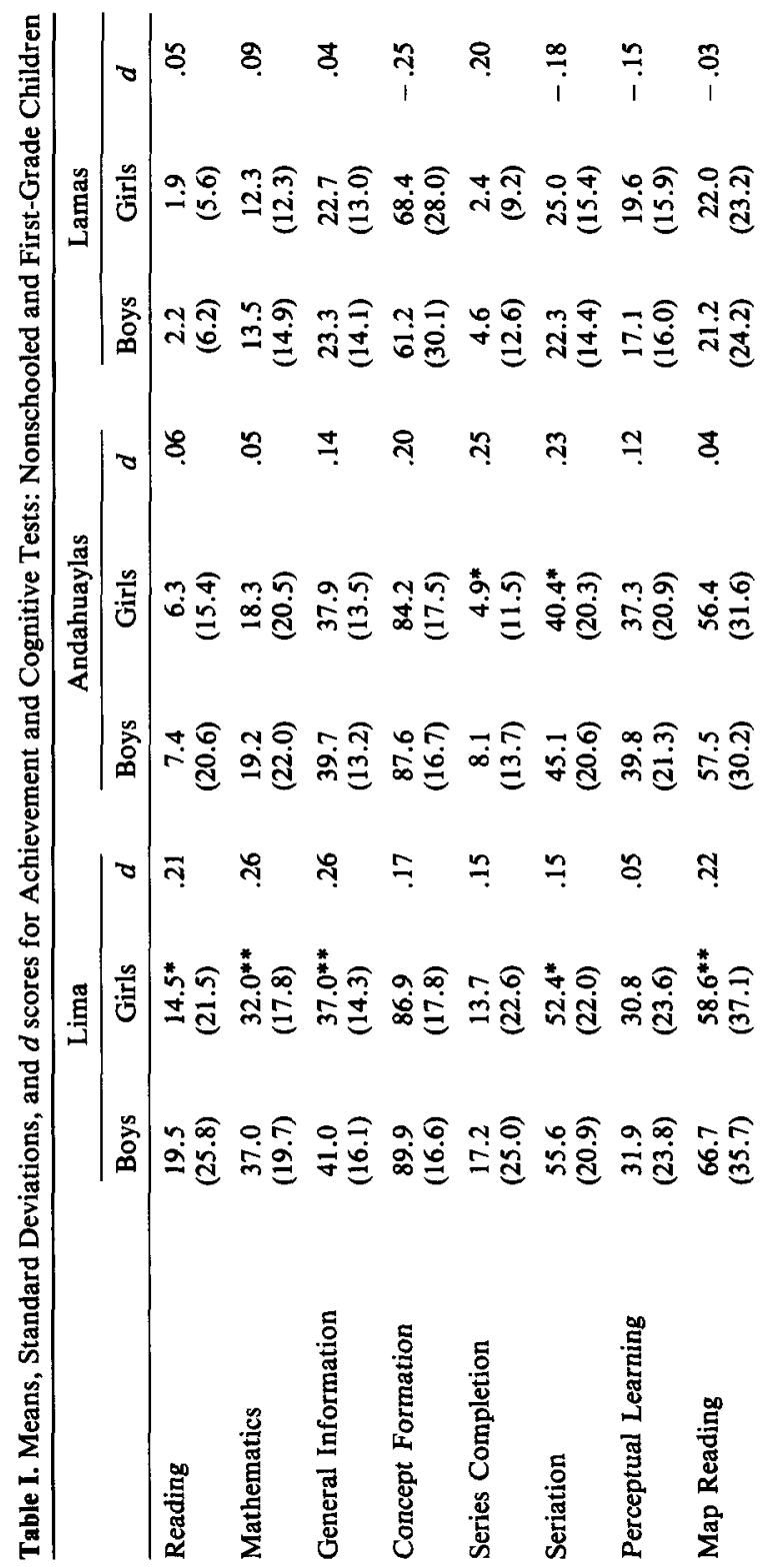




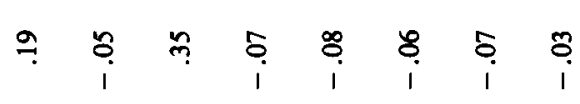

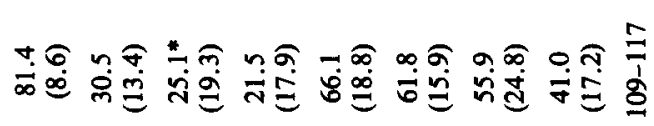

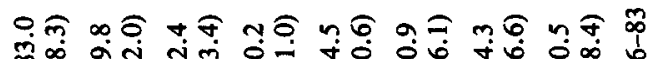
ळळ



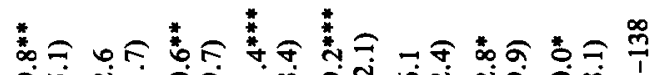


Пกิต

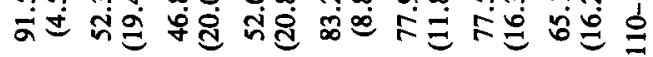
मे ప

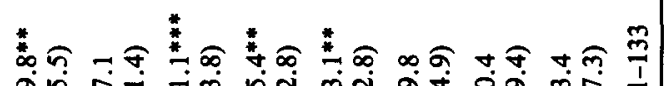

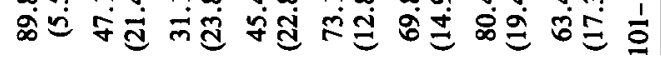

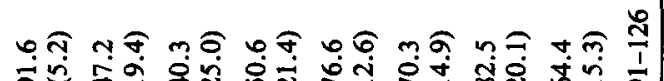

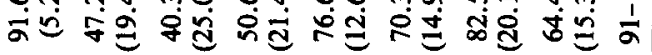

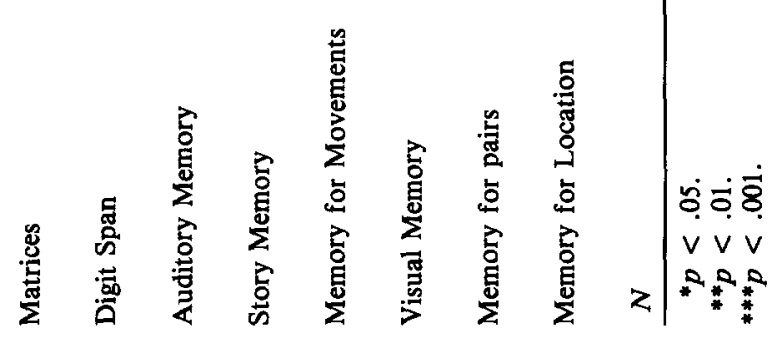


Table II. Summary Table of ANOVAS of Gender (G) Effects and Interactions with Location (L), Age (A), and Schooling (S)

\begin{tabular}{|c|c|c|}
\hline & $\begin{array}{l}\text { Nonschooled and } \\
\text { Grade } 1\end{array}$ & Grades $1,2,3$ \\
\hline Reading & $G \times A \times S^{*}$ & ns \\
\hline Mathematics & $\mathrm{G}^{*}$ & ns \\
\hline General Information & $G, * * G \times L \times A \times S^{*}$ & $\mathrm{G}^{*}$ \\
\hline Concept Formation & & ns \\
\hline Series Completion & $\mathrm{G}^{* *}$ & ns \\
\hline Seriation & $\mathrm{G}^{*}$ & ns \\
\hline Perceptual Learning & ns & ns \\
\hline Map Reading & $\mathrm{G},{ }^{*} \mathrm{G} \times \mathrm{L}^{*}$ & ns \\
\hline Matrices & $\mathrm{G}^{* * *}$ & $\mathrm{G},{ }^{* *} \mathrm{G} \times \mathrm{S},{ }^{*} \mathrm{G} \times \mathrm{L} \times \mathrm{S}^{*}$ \\
\hline Digit Span & ns & ns \\
\hline Auditory Memory & $\mathrm{G}, * * * \mathrm{G} \times A \times \mathrm{S}^{*}$ & $\mathrm{G}^{* * *}$ \\
\hline Story Memory & $G, * * * G \times A^{* *}$ & $G, * * G \times S^{*}$ \\
\hline Memory for Movements & $\mathrm{G}^{* *}$ & $\mathrm{G}, * \mathrm{G} \times \mathrm{S}^{*}$ \\
\hline Visual Memory & & ns \\
\hline Memory for Pairs & $\mathrm{G},{ }^{*} \mathrm{G} \times \mathrm{L} \times \mathrm{A}^{*}$ & $G \times S^{*}$ \\
\hline Memory for Location & $\mathrm{G}^{*}$ & ns \\
\hline
\end{tabular}

${ }^{*} p<.05$.

${ }^{* *} p<.01$

${ }^{* * *} p<.001$.

and age group) were entered with gender in regression analyses of each task. For the "older" first through third graders, the variable of age was dropped.

The results of the analyses are summarized in Table IV. For the nonschooled and first graders, the background variables accounted for from 12 to $43 \%$ of the variance, depending upon the task; for the "older" first through third graders, the percentage ranged from 6 to 39\%. In contrast, gender never accounted for more than around 3 or $4 \%$ of the variance. Within these small percentages, the degree of influence of gender depended on the type of task under consideration.

\section{DISCUSSION}

These results provide little support to those who argue that boys possess greater quantitative and spatial skills than girls and that girls have greater verbal abilities than boys (Maccoby \& Jacklin, 1974) but are in line with the conclusion drawn from the meta-analyses of Hyde and Linn (1988) and of Hyde et al. (1990) that knowing a child's gender adds little to one's ability to predict scores on cognitive or achievement tests. At the same time, two important constraints on this conclusion did emerge. First, 
the child's gender exerted a more significant effect for some tasks than for others. Some of the strongest effects were found for General Information, Auditory Memory, and Story Memory. On the other hand, knowing the child's gender did nothing to improve the prediction of scores on Reading, Concept Formation, Perceptual Learning, Digit Span, or Visual Memory beyond that resulting from consideration of other background variables.

We have no ready explanation why the gender effects were greater for some tasks than for others. It does not seem likely that they were due to methodological factors, such as reliability, order effects, or rapport. The tasks were reliable, they were given in different orders to different subjects, and rapport with the subjects was excellent throughout testing. The most reasonable interpretation is that there are small, but significant gender differences in cognitive functioning that were tapped by the different tests. Such differences merit continued exploration, especially in studies where an array of different tasks is given to the same subjects. Through this procedure it is possible to avoid any confounding effects of populations sampled and cognitive tasks employed, thereby reducing the difficulty of interpreting inconsistent effects that may arise in comparing studies involving differences both in tasks and in populations.

A second constraint on generalizations about the effects the influences of gender lies in the interactive effects of schooling and gender. Gender effects tended to decline with increasing amounts of schooling. Moreover, when the gender by schooling interaction was significant, the effect represented smaller gender differences in scores with increased amounts of schooling. These findings suggest that as boys and girls share common experiences through their schooling, differences in their cognitive functioning, as well as in their academic achievement, decrease. This was the case for elementary school children. Whether it also would be the case for secondary school students is not clear.

The tendency for boys to obtain higher scores than girls is related, we believe, to the privileged status of boys in Andean cultures. We had expected gender effects might differ according to urban-rural residence, but this did not occur. Rather, there were indications of greater gender effects in Lima and Andahuaylas than in Lamas. Although the analyses of variance revealed few interactions between location and gender when location was included as a variable, there were indications in separate analyses for each location and in the $d$ scores that the departures were greater between Lamas and the other two locations than between the city and the countryside.

Interviews with the children's mothers offered evidence of the differential status of boys and girls in the three locations. The mothers told us that the fathers participated in cognitive activities with boys more frequent- 


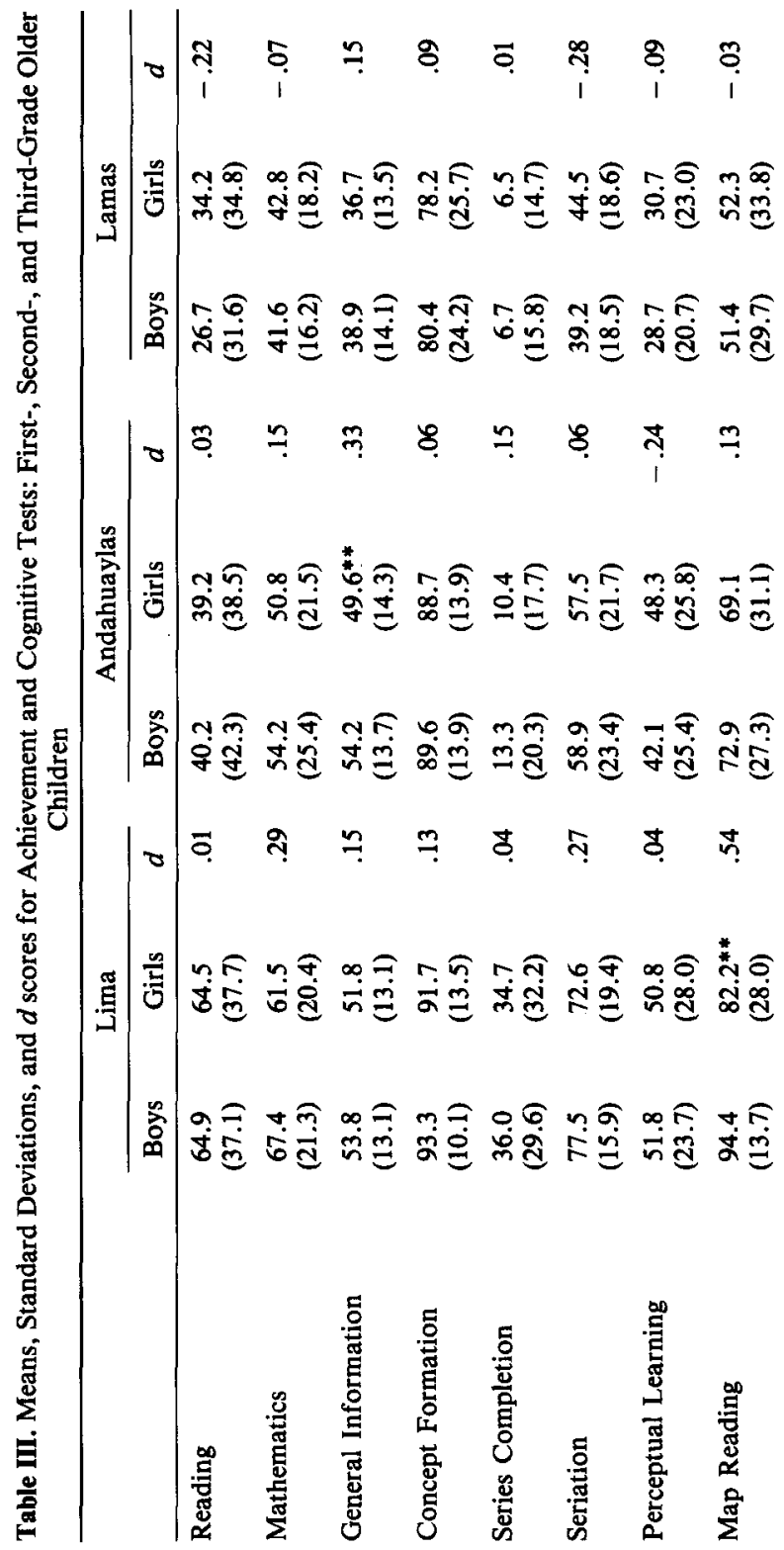




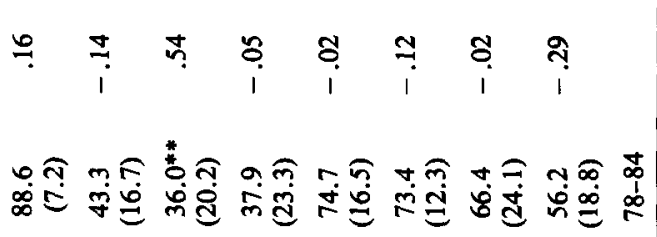

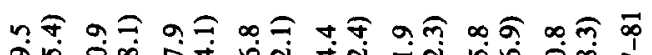

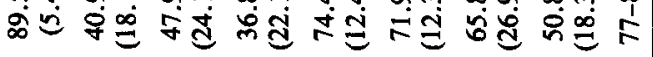

ธำ ๆ ๆ

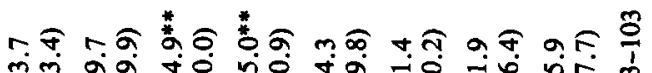
के

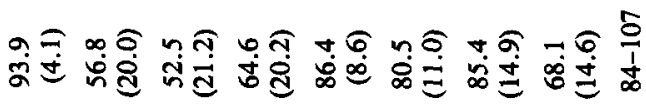

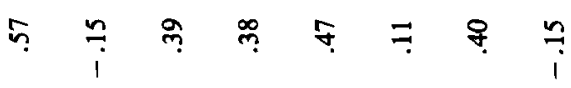

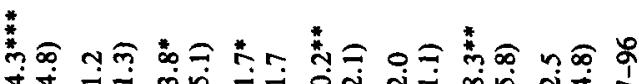
过过过

ทศิจ

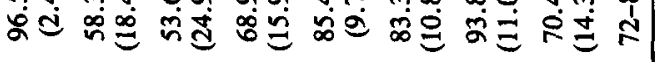

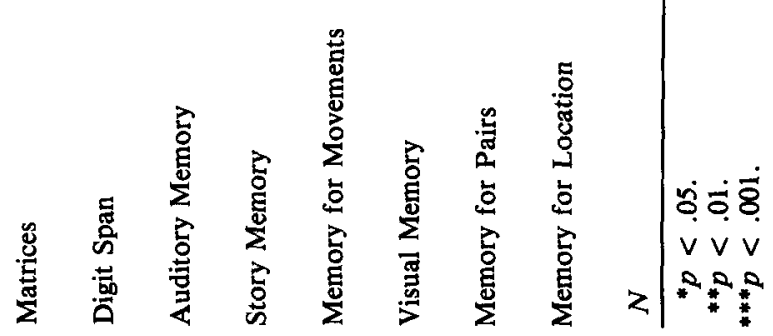


Table IV. Percentage of Variance Contributed by Background Variables and Gender for Each Task

\begin{tabular}{|c|c|c|c|c|}
\hline & \multicolumn{2}{|c|}{$\begin{array}{l}\text { Nonschooled and } \\
\text { Grade } 1\end{array}$} & \multicolumn{2}{|c|}{ Grades $1,2,3$} \\
\hline & $\begin{array}{c}\text { Background } \\
\text { variables }\end{array}$ & Gender & $\begin{array}{l}\text { Background } \\
\text { variables }\end{array}$ & Gender \\
\hline Reading & 22.6 & 0.3 & 36.6 & 0.1 \\
\hline Mathematics & 43.8 & $0.4^{*}$ & 39.4 & 0.3 \\
\hline General Information & 34.9 & $0.9^{* *}$ & 27.6 & $0.9^{* *}$ \\
\hline Concept Formation & 20.7 & 0.0 & 8.8 & 0.2 \\
\hline Series Completion & 11.7 & $0.9^{* *}$ & 27.0 & 0.1 \\
\hline Seriation & 38.2 & $0.5 *$ & 37.7 & 0.0 \\
\hline Perceptual Learning & 16.7 & 0.1 & 19.1 & 0.3 \\
\hline Map Reading & 33.6 & $0.6^{*}$ & 27.7 & 0.5 \\
\hline Matrices & 34.8 & $1.7^{* * *}$ & 29.3 & $1.0^{*}$ \\
\hline Digit Span & 21.5 & 0.0 & 19.9 & 0.5 \\
\hline Auditory Memory & 11.5 & $3.1^{* * *}$ & 5.5 & $4.2^{* * *}$ \\
\hline Story Memory & 32.6 & $1.8^{* * *}$ & 27.7 & $1.3^{* *}$ \\
\hline Memory for Movements & 23.3 & $0.8^{* *}$ & 13.8 & $0.9^{*}$ \\
\hline Visual Memory & 25.1 & 0.2 & 16.3 & 0.0 \\
\hline Memory for Pairs & 26.5 & $0.4^{*}$ & 23.8 & 0.4 \\
\hline Memory for Location & 30.2 & $0.5^{*}$ & 19.3 & 0.2 \\
\hline$N \mathrm{~s}$ & \multicolumn{2}{|c|}{$619-740$} & \multicolumn{2}{|c|}{$471-555$} \\
\hline
\end{tabular}

Note. Background variables included location, age groups, and years of schooling. Percentages of variance accounted for by background variables are all statistically significant, $p$ 's $<.001$.

${ }^{*} p<.05$.

$* * p<.01$.

${ }^{* * *} p<.001$

ly than girls. The mean numbers of activities were 1.4 and .9 for boys and girls in Lima, 1.4 and 1.1 in Andahuaylas, and 2.5 and 2.4 in Lamas. (We asked about five activities: teaching about colors, counting, and letters; reading to the child; and telling stories to the child.) Another tendency mentioned by the mothers was for girls to be taught more practical activities by their mothers. In addition, boys were expected to use money earlier than girls, to stay alone a whole day, and to take a half-day trip, but girls were expected to be able to take care of their siblings at a younger age than boys. Boys were expected to attain jobs and a position in the community that were of a higher status than those attained by girls. The smallness of the gender effects found in the various tests become even more impressive in view of these indications that parents provided boys with greater opportunities for cognitive stimulation than they provided girls.

Our search of the literature for research about the influence of schooling on gender differences in cognitive ability of young children revealed no formal studies, thus we cannot evaluate the generality of the tendencies that we found for the differences between the scores of boys and girls to decline 
with increases in amount of schooling. We will have a better grasp of how culture and gender interact when we have more information about how attending school influences memory, perception, learning, and other cognitive functions of boys and girls.

\section{REFERENCES}

Feingold, A. Cognitive gender differences are disappearing. American Psychologist, 1988, 43, 93-103.

Frisancho, A. R., Borkan, G. A., \& Klayman, J. E. Pattern of growth of lowland and highland Peruvian Quechua of similar genetic composition. Human Biology, 1975, 47, 233-243.

Hanna, G. Gender differences in mathematics achievement among eighth graders: Results from twenty countries. In L. Tighe (Chair), Gender and cognitive skills: Cross-cultural and ecological perspectives. Symposium conducted at the meeting of the American Association for the Advancement of Science, Boston, Jan. 1988.

Harnisch, D. L. Females and mathematics: A cross-national perspective. In M. Maehr (Ed.), Advances in motivation and achievement (Volume 2, pp. 73-91). Greenwich, CT: JAI Press, 1986.

Harnisch, D. L., Steinkamp, M. W., Tsai, S. L., \& Walberg, H. J. Cross-national differences in mathematics attitude and achievement among seventeen-year-olds. International Journal of Educational Development, 1986, 6, 233-244.

Hedges, L. V., \& Becker, B. J. Statistical methods in the meta-analysis of research on gender differences. In J. S. Hyde \& M. C. Linn (Eds.), The psychology of gender: Advances through meta-analysis (pp. 14-50). Baltimore: The Johns Hopkins University Press, 1986.

Hyde, J. A., \& Linn, M. C. Gender differences in verbal ability: A meta-analysis. Psychological Bulletin, 1988, 104, 53-69.

Hyde, J. S., Fennema, E., \& Lamon, S. J. Gender differences in mathematics performance: A meta-analysis. Psychological Bulletin, 1990, 107, 139-155.

Linn, M. C., \& Hyde, J. S. Gender, mathematics, and science. Educational Researcher, 1989, $18,17-27$.

Lummis, M., \& Stevenson, H. W. Gender differences in beliefs and achievement: A crosscultural study. Developmental Psychology, 1990, 26, 254-263.

Maccoby, E., \& Jacklin, C. The psychology of sex differences. Stanford, CA: Stanford University Press, 1974.

Rosenblatt, P. C., \& Cunningham, M. R. Sex differences in cross-cultural perspective. In B. Lloyd \& J. Archer (Eds.), Exploring sex differences (pp. 71-94). New York: Academic Press, 1976.

Stevenson, H. W., Parker, T., Wilkinson, A., Hegion, A., \& Fish, E. Predictive value of teachers' ratings of young children. Journal of Educational Psychology, 1976, 68, $507-517$.

Stevenson, H. W., Stigler, J. W., Lee, S. Y., Lucker, G. W., Kitamura, S., \& Hsu, C. C. Cognitive performance and academic achievement of Japanese, Chinese, and American children. Child Development, 1985, 56, 718-734. 